\title{
Effectiveness of pregabalin for the treatment of chronic low back pain with accompanying lower limb pain (neuropathic component): a non-interventional study in Japan
}

\author{
This article was published in the following Dove Press journal: \\ Journal of Pain Research \\ 5 August 2015 \\ Number of times this article has been viewed
}

\author{
Toshihiko Taguchi ${ }^{1}$ \\ Ataru Igarashi \\ Stephen Watt ${ }^{3}$ \\ Bruce Parsons ${ }^{3}$ \\ Alesia Sadosky ${ }^{3}$ \\ Kazutaka Nozawa ${ }^{4}$ \\ Kazuhiro Hayakawa ${ }^{4}$ \\ Tamotsu Yoshiyama ${ }^{4}$ \\ Nozomi Ebata ${ }^{4}$ \\ Koichi Fujii ${ }^{4}$ \\ 'Department of Orthopaedic Surgery, \\ Yamaguchi University Graduate \\ School of Medicine, Ube, Yamaguchi, \\ Japan; '2Department of Drug Policy \\ and Management, Graduate School \\ of Pharmaceutical Sciences, The \\ University of Tokyo, Tokyo, Japan; \\ ${ }^{3}$ Pfizer Inc., New York, NY, USA; \\ ${ }^{4}$ Pfizer Japan, Inc., Tokyo, Japan
}

Objective: To evaluate the impact of pregabalin on sleep, pain, function, and health status in patients with chronic low back pain with accompanying neuropathic pain (CLBP-NeP) under routine clinical practice.

Methods: This prospective, non-interventional, observational study enrolled Japanese adults ( $\geq 18$ years) with CLBP-NeP of duration $\geq 3$ months and severity $\geq 5$ on a numerical rating scale $(0=$ no pain, $10=$ worst possible pain). Treatment was 8 weeks with pregabalin $(n=157)$ or usual care alone $(n=174)$; choice of treatment was determined by the physician. The primary efficacy outcome was change from baseline to 8 weeks in pain-related interference with sleep, assessed using the Pain-Related Sleep Interference Scale (PRSIS; 0= did not interfere with sleep, $10=$ completely interferes with sleep). Secondary endpoints were changes in PRSIS at week 4, and changes at weeks 4 and 8 in pain (numerical rating scale), function (Roland-Morris Disability Questionnaire), and quality of life (EuroQol 5D-5L); global assessments of change were evaluated from the clinician and patient perspectives at the final visit.

Results: Demographic characteristics were similar between cohorts, but clinical characteristics suggested greater disease severity in the pregabalin group including a higher mean (standard deviation) pain score, $6.3(1.2)$ versus $5.8(1.1)(P<0.001)$. For the primary endpoint, pregabalin resulted in significantly greater improvements in PRSIS at week 8 , least-squares mean changes of -1.3 versus -0.4 for usual care $(P<0.001)$; pregabalin also resulted in greater PRSIS improvement at week $4(P=0.012)$. Relative to usual care at week 8 , pregabalin improved pain and function (both $P<0.001$ ), and showed global improvements since beginning study medication $(P<0.001)$. Pregabalin was well tolerated.

Conclusion: In clinical practice in patients with CLBP-NeP, pregabalin showed significantly greater improvements in pain-related interference with sleep relative to usual care. In addition, pregabalin significantly improved pain, function, and health status, suggesting the benefits of pregabalin for overall health and well-being relative to usual care in these patients. (Clinicaltrials. gov identifier NCT02273908).

Keywords: pregabalin, usual care, chronic low back pain, neuropathic pain, non-interventional study, sleep

\section{Introduction}

Low back pain (LBP) is one of the most common major health problems in industrialized countries, and is ranked among the top ten conditions that account for the highest number of disability-adjusted life years worldwide. ${ }^{1}$ People of all ages can be affected by LBP, and because of its widespread prevalence it is one of the most burdensome
Correspondence: Alesia Sadosky Pfizer Inc., 235 East 42nd Street, New York, NY 10017 , USA

Tel + I 212733 949I

Fax + I 646 44I 4757

Email alesia.sadosky@pfizer.com 
pain conditions. This burden results from its association with high health care resource utilization, and reductions in function and work productivity. 2,3

In Japan, the estimated lifetime risk of LBP is $83 \%,{ }^{4}$ and its presence also results in reductions in quality of life. ${ }^{5}$ Furthermore, as evidenced by a recent study of patients with LBP in Japan, pain severity in LBP is associated with an increased patient burden, with a greater burden at higher levels of pain. ${ }^{6}$ In particular, patients reported significantly greater sleep disturbance and impairment of quality of life at higher pain severity levels. ${ }^{6}$

When LBP lasts $\geq 3$ months it is known as chronic LBP (CLBP), and this chronicity significantly increases the burden relative to that of LBP of shorter duration. ${ }^{7}$ CLBP can be considered a mix of three pain components depending on the presentation: nociceptive, neuropathic origin, or augmented central pain processing. ${ }^{8,9}$ In the context of CLBP, the most common syndrome is neuropathic pain $(\mathrm{NeP})$ secondary to nerve root involvement. ${ }^{10,11}$

In Japan, it has been estimated that $29.4 \%$ of patients with CLBP have an NeP component (CLBP-NeP), ${ }^{12}$ which is consistent with the rate of $37.0 \%$ that has been reported in a European population ${ }^{13}$ and estimated in an evidence-based review. ${ }^{14}$ A post-marketing surveillance study further identified radiculopathy including CLBP (ie, CLBP-NeP) as the most prevalent NeP condition in Japan, accounting for $40 \%$ of patients with NeP. ${ }^{15}$

Sleep is an especially important outcome that affects daily function and quality of life, and there appears to be a reciprocal relationship between chronic pain and sleep in chronic pain conditions. ${ }^{16,17}$ This relationship includes the potential for sleep disturbance to lower the pain threshold. ${ }^{18}$ Furthermore, a systematic review of the relationships between CLBP and sleep reported that CLBP negatively affects multiple sleep dimensions, including reductions in sleep duration and quality, and poor daytime functioning. ${ }^{19}$

Sleep disturbance is one of the most important coexisting conditions in patients with CLBP, ${ }^{20,21}$ and similarly in NeP, with real-world studies revealing that sleep disturbances are the most frequently patient-reported effects of NeP. ${ }^{22,23} \mathrm{As}$ in other NeP conditions, patients with CLBP-NeP report high pain severity, which is associated with poor sleep as well as reduced function and productivity, ${ }^{24}$ sleep disturbance is a comorbid condition that is reported in at least $50 \%$ of these patients with CLBP-NeP regardless of pain severity. Indeed, the presence of NeP in CLBP also appears to exacerbate sleep problems, as suggested by poorer sleep outcomes (ie, more frequent disturbance, greater reductions in quality and adequacy, and increased daytime somnolence) than patients with CLBP less likely to have an NeP component. ${ }^{13}$ Both the pain and the sleep disturbance affect the ability to function and perform daily activities. Functional ability in CLBP is frequently measured using the condition-specific Roland-Morris Disability Questionnaire (RMDQ), ${ }^{25}$ and together, these outcomes of pain, sleep, and function can be considered integral components that contribute to the health state of the individual and the overall efficacy of CLBP treatment. ${ }^{26}$ Indeed, contemporary evidence-based NeP guidelines highlight taking into account pain severity, and its impact on lifestyle and daily activities including sleep disturbance, when discussing an appropriate management strategy with a patient. ${ }^{27}$

Treatment guidelines have been developed for CLBP, and although non-pharmacologic strategies are used, management primarily relies on a pharmacologic approach. ${ }^{28}$ However, the presence of $\mathrm{NeP}$ increases the challenge of treatment, since many of the standard analgesics recommended in these guidelines, such as acetaminophen and nonsteroidal anti-inflammatory drugs, have poor efficacy for NeP. This greater complexity in treating NeP provided the rationale for development of specific recommendations for NeP management. ${ }^{29}$

Pregabalin, a high affinity ligand of alpha-2-delta $(\alpha 2-\delta)$ subunits of voltage-gated calcium channels in the central nervous system, ${ }^{30}$ is considered a first-line drug for the treatment of NeP. ${ }^{29}$ It has received Japanese manufacturing and marketing approval to treat NeP based on its demonstrated efficacy in clinical trials for reducing pain in patients with different NeP diseases including post-herpetic neuralgia, diabetic peripheral neuropathy, and NeP associated with spinal cord injury. ${ }^{31-33}$ Its safety profile in Japanese patients has also been shown to be similar to that observed in western studies. $^{34}$

In randomized clinical trials, pregabalin significantly improved patient-reported sleep disturbance across multiple conditions, through an indirect analgesic effect and a direct effect on sleep. ${ }^{35}$ Observational studies from routine clinical practice in primary care settings in Germany and Spain in which analgesic therapy was based on the clinical judgment of the treating physician have also shown that pregabalin improves pain and sleep disturbance when used for the treatment of CLBP with an NeP component. ${ }^{36,37}$ However, the practical therapeutic value of pregabalin relative to other pain medications has not been assessed in CLBP-NeP patients in a Japanese typical care setting. Therefore, since it is important to demonstrate generalizability of results across 
populations, ${ }^{38}$ the goal of this non-interventional study is to evaluate from the patient's perspective the benefits of usual care with and without pregabalin for the treatment of CLBP with NeP in Japanese patients in a variety of clinical settings. The impact of pregabalin was evaluated on sleep interference, pain, function, and impression of change at end of treatment. In particular, the effects on sleep were evaluated as the primary endpoint, since it is ranked of high importance among pain-related outcomes across a variety of chronic pain conditions, ${ }^{39}$ and thus would represent a true benefit from the patient's perspective. Furthermore, based on the importance of sleep, guidelines for the assessment of chronic pain as well as guidelines specific to $\mathrm{NeP}$ consistently emphasize sleep as a core outcome domain recommended for assessment in clinical trials. ${ }^{40-42}$

\section{Methods \\ Study design}

This 8-week, prospective, non-interventional, observational study, was performed at 33 study sites in Japan. All sites regularly treat patients with CLBP accompanied by NeP in a primary care setting using routine clinical practice. The study was conducted in the latter half of 2014, and study sites encompassed a variety of clinical settings including orthopedic surgery (28), pain (two), internal medicine (one), urology (one), and general surgery (one). These study sites were screened and selected based only on feasibility from 6,428 medical institutions that have contracted with a site management organization and have accepted support by clinical research coordinators across Japan. The present study was designed to evaluate the effectiveness of pregabalin versus usual care for the treatment of CLBP with an NeP component in primary care settings. Effectiveness was measured from the patient's perspective using several patient-completed measures. The study was performed in accordance with the Declaration of Helsinki and received approval from the Byoin-Godo Ethical Review Board; all patients provided written informed consent prior to participation (Clinicaltrials.gov identifier NCT02273908).

Patients who enrolled in the study were treated for 8 weeks with usual care, defined as conventional analgesic care, or pregabalin alone or in addition to whatever other analgesics they may have been prescribed, with choice of therapy determined solely by the clinical judgment of the treating physician.

\section{Patient population}

For inclusion, Japanese adults ( $\geq 18$ years old) were required to have CLBP with accompanying lower limb pain below the knee of duration $\geq 3$ months that was also refractory to previous analgesics for $\geq 3$ months, and of severity $\geq 5$ (at least moderate severity) on a numerical rating scale (NRS; $0=$ no pain, $10=$ worst possible pain). Patients who were treated with pregabalin within the past 2 weeks were excluded from the study. All patients who were encountered during the enrollment period and met all study criteria were continuously enrolled at the study sites in order to reduce selection bias. The self-report version of the Leeds Assessment of Neuropathic Symptoms and Signs pain scale ${ }^{43}$ was administered after the physical exam but was not used for diagnosis.

\section{Outcomes}

The primary efficacy endpoint was change from baseline at 8 weeks in the Pain-Related Sleep Interference Scale (PRSIS) with a recall period over the past week. Sleep interference was used as the primary efficacy endpoint since it is not only an outcome of importance to patients, but has a reciprocal relationship with pain, ${ }^{16,17,44-47}$ and CLBP has been reported to significantly affect quality of sleep..$^{20,48}$ The PRSIS is a patient-completed measure that evaluates the extent to which pain interferes with sleep, and is scored using an NRS ranging from 0 ("did not interfere with sleep") to 10 ("completely interferes with sleep"). It is derived from the Pain Interference with Sleep item of the Brief Pain Inventory-Short Form ${ }^{49}$ and except for the 1-week recall period, it is similar to the Daily Sleep Interference Scale that has been validated to evaluate sleep interference due to pain in patients with NeP. ${ }^{50}$ The PRSIS format has been used previously in almost all clinical studies of pregabalin in $\mathrm{NeP},{ }^{51,52}$ and in the current study it was administered at baseline, and after 4 and 8 weeks of treatment.

Secondary endpoints included changes in pain and function from baseline to weeks 4 and 8; subjects were asked to describe their average pain over the past week, assessed using the NRS, and function was assessed using the RMDQ,${ }^{25}$ as recommended by the Japan Orthopaedic Association's guidelines for the management of $\mathrm{LBP}^{26}$ In the event that a patient had used rescue medication within 12 hours of the clinic visit, the patient was requested to respond to the RMDQ questions based on their functional ability for the 24-hour period preceding the rescue medication. The RMDQ is a patient-completed measure that evaluates how well patients with CLBP function with regard to daily activities during the previous 24-hour period. The range of scores on the RMDQ is from 0 to 24 , and lower scores indicate better function. While a 3.5-5-point absolute change has been considered 
as the minimal clinically important difference, ${ }^{53,54}$ a $30 \%$ change from baseline is generally accepted, since this value provides an estimate of clinical relevance regardless of baseline score..$^{54,55}$

The proportions of patients who achieved improvements in pain of $30 \%$ and $50 \%$ were also determined, as recommended by the Initiative on Methods, Measurement, and Pain Assessment in Clinical Trials (IMMPACT) ${ }^{56} \geq 30 \%$ is considered a clinically significant pain reduction, and a reduction $\geq 50 \%$ is of substantial clinical significance.

Other secondary endpoints were the 5-level, 5 dimension EuroQol (EQ-5D-5L), ${ }^{57}$ and global impression of change assessed by both the patient and clinician, using the Patient Global Impression of Change (PGI-C) and Clinician Global Impression of Change (CGI-C), respectively. ${ }^{58}$ The EQ-5D-5L measures included the change from baseline in current health state, with "today" as the recall period, on the visual analog scale (VAS) health state thermometer standardized to a 0-100 scale, and the change in quality of life score using the Japanese value set. ${ }^{59}$ Validated Japanese versions of all measures were used, including the RMDQ. ${ }^{60}$

The primary objectives of this study did not include comparison of safety with usual care, consistent with the non-interventional nature of the study.$^{61}$ However, safety information for pregabalin was collected in accordance with the standard practice of the sponsor, and was evaluated based on the incidence of treatment-related adverse events (AEs) using a safety analysis set, defined as all subjects who received at least one dose of pregabalin.

\section{Statistical analysis}

Sample size was determined based on the proposed analysis of the primary endpoints. Assumptions for calculation of sample size, derived from previous Japanese studies of pregabalin for $\mathrm{NeP}$ and a post-marketing study that is currently ongoing in Japan, included a standardized effect size of 0.35 (ie, the difference in mean change from baseline divided by the pooled standard deviation of the mean change from baseline); a $25 \%$ withdrawal rate; correlation of 0.85 for scores between weeks 4 and 8 ; and a ratio of pregabalin to usual care of $1: 1$. Based on these assumptions, it was estimated that a total sample size of 324 subjects would be needed to have at least $80 \%$ power to detect the treatment difference.

Descriptive and inferential statistical analyses were performed. Efficacy analyses were conducted on the full analysis set, which included all patients who received at least one dose of the pregabalin or usual care treatment and had at least one post-baseline evaluable efficacy assessment. The PRSIS, pain NRS, RMDQ, and EQ-5D-5L were summarized at baseline, and for the change from baseline at weeks 4 and 8; the PGI-C and CGI-C were summarized at the end of the treatment period. The primary endpoint was defined as change from baseline to week 8 in the PRSIS, and treatment groups were compared using a mixed model for repeated measures analysis ${ }^{62}$ that included fixed categorical effects of treatment, visit, and treatment-by-visit interaction, as well as a fixed continuous effect of baseline value. Significance was declared if the 2-tailed test for the difference between treatment groups was significant at the 0.05 level. No adjustments were made for multiplicity, since all comparisons, except for a single primary comparison, were considered secondary. The same mixed model for repeated measures was used for all other analyses of continuous data. Responders of pain NRS were analyzed using a logistic regression model that included treatment and baseline value, and chi-square test was used for PGI-C and CGI-C.

A post hoc sensitivity analysis of the PRSIS at week 8/endpoint was performed in which missing values were imputed using baseline observation carried forward (BOCF) on all treated subjects regardless of whether a patient had at least one post-baseline evaluable score of PRSIS.

The statistical analysis plan was developed before any data became available, and all analyses were conducted using SAS version 9.2 (SAS Institute Inc., Cary, NC, USA).

\section{Results}

\section{Patient characteristics}

A total of 331 patients were enrolled and 293 completed the study, $79.0 \%$ and $97.1 \%$ of the pregabalin and usual care groups, respectively (Figure 1); the primary reasons for discontinuation were AEs (10.2\%) and lost to follow-up (6.4\%) in the pregabalin group, and patient request in the usual care group (1.1\%). Baseline demographics (Table 1) were similar between the groups; the population was predominantly female $(59.8 \%)$, with a mean (standard deviation) age of 70.0 (13.1) years. Distribution of the primary diagnoses (Table 1) was also similar between groups, and the two main pain diagnoses were lumbar spinal stenosis (34.1\%) and osteoarthritis of the lumbar spine (32.6\%). The mean baseline pain NRS in the pregabalin group, $6.3(1.2)$, was significantly higher than that in the usual care group 5.8 (1.1), $(P<0.001)$, as was the RMDQ score (10.6 [5.2] versus 9.0 [4.9]; $P=0.006)$, although the baseline PRSIS scores were comparable between the treatment groups, 3.1 (2.7) and 2.9 (2.4), respectively $(P=0.409)$. 


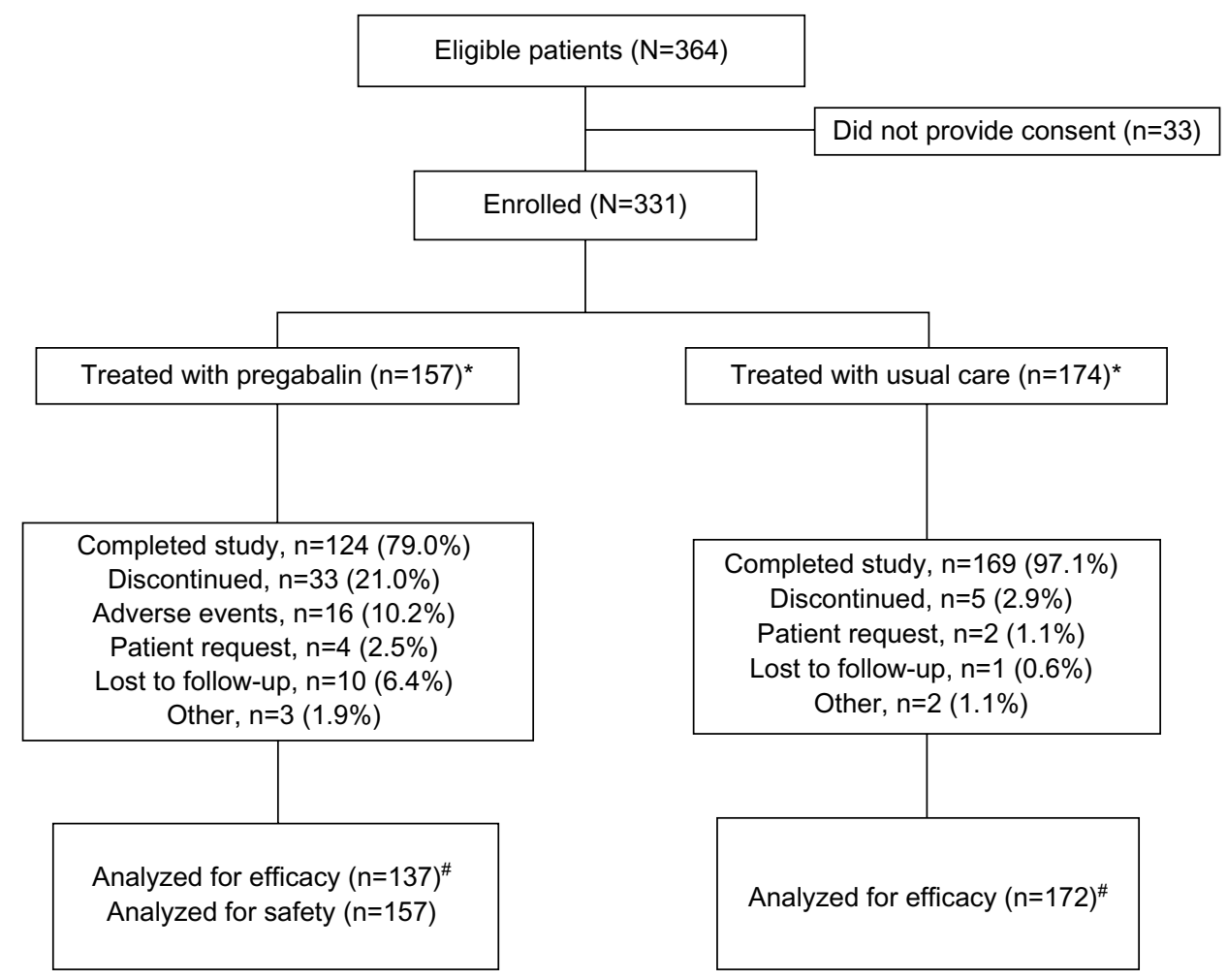

Figure I Patient disposition.

Notes: *Choice of therapy determined by the clinical judgment of the treating physician; "the full analysis set consisted of subjects who had at least one evaluable observation from any of the patient-reported outcomes, and only evaluable subjects who contributed to the particular outcome were evaluated in each analysis.

All patients used a variety of medications for pain (Table 1). Nonsteroidal anti-inflammatory drugs were the most commonly reported analgesics, used by $96.8 \%$ and $95.4 \%$ of pregabalin and usual care patients, respectively, and there was polypharmacy in both treatment arms. No patients were enrolled who had been treated with pregabalin during the 3 months prior to the study.

\section{Efficacy}

Pregabalin dosing was flexible, and the range of doses among these patients was $25 \mathrm{mg} /$ day to $300 \mathrm{mg} /$ day. For the primary efficacy endpoint of PRSIS at week 8 , patients treated with pregabalin reported a least-squares (LS) mean change from baseline of -1.3 compared with -0.4 for usual care (Figure 2). These changes resulted in a treatment difference of $-0.9(95 \%$ confidence interval $[\mathrm{CI}]-1.5,-0.4)$ that significantly favored pregabalin $(P<0.001)$. Significant differentiation between treatments for improvement in PRSIS was also observed at week 4 (Figure 2), with a difference in LS mean PRSIS scores of -0.7 (95\% CI $-1.2,-0.1 ; P=0.012)$ indicating greater improvement with pregabalin.

As shown in Figure 3A, pregabalin resulted in a significantly greater improvement in pain relative to usual care at week 4 , with LS mean change from baseline of -1.8 compared with -1.1 for the usual care (difference in LS mean -0.7 [95\% CI -1.1, -0.2]; $P=0.004)$. Additional improvement was observed at week 8 , which resulted in a between-treatment difference in LS mean change from baseline of -0.8 (95\% CI $-1.3,-0.4 ; P<0.001)$ that favored pregabalin. Compared with usual care, significantly greater proportions of pregabalin-treated patients achieved clinically relevant reductions in pain of $\geq 30 \%$ (54.6\% versus $37.1 \% ; P=0.008$ ) and $\geq 50 \%$ (39.2\% versus 20.0\%; $P=0.002$ ) (Figure 3B).

Patient-reported function was significantly improved in the pregabalin group relative to usual care as indicated by greater reductions from baseline in RMDQ scores at weeks 4 and 8 (both $P<0.001$ ) (Figure 4), representing changes of $29 \%$ and $38 \%$, respectively. While patients treated with pregabalin also reported greater improvements in EQ-5D-5L VAS health state at both time points, the difference between treatment groups was only significant at week 8 , with an LS mean change of 7.0 versus 2.3 for usual care $(P=0.026)$, resulting in a difference in LS mean of $4.7(95 \% \mathrm{CI} 0.6,8.8)$. In contrast, the EQ-5D-5L quality of life score showed a significant improvement favoring pregabalin at week 4 , with an LS mean difference in scores of 0.0329 (95\% CI 0.0058, 
Table I Baseline demographic and clinical characteristics of the treatment groups

\begin{tabular}{|c|c|c|c|}
\hline Variable & $\begin{array}{l}\text { Pregabalin } \\
(\mathrm{N}=157)\end{array}$ & $\begin{array}{l}\text { Usual care } \\
(\mathrm{N}=174)\end{array}$ & $P$-value ${ }^{a}$ \\
\hline Sex, n (\%) & & & 0.667 \\
\hline Male & $65(4 I .4)$ & $68(39.1)$ & \\
\hline Female & $92(58.6)$ & $106(60.9)$ & \\
\hline Age, years, mean \pm SD & $69.1 \pm 14.1$ & $70.7 \pm 12.2$ & 0.272 \\
\hline Age distribution, n (\%) & & & 0.564 \\
\hline$<65$ years & $43(27.4)$ & $44(25.3)$ & \\
\hline $65-74$ years & $50(31.8)$ & $49(28.2)$ & \\
\hline$\geq 75$ years & $64(40.8)$ & 81 (46.6) & \\
\hline $\begin{array}{l}\text { Primary pain diagnosis, } \\
\mathrm{n}(\%)\end{array}$ & & & 0.258 \\
\hline Lumbar disc herniation & $22(14.0)$ & $17(9.8)$ & \\
\hline Lumbar spinal stenosis & $49(3 \mid .2)$ & $64(36.8)$ & \\
\hline $\begin{array}{l}\text { Osteoarthritis of } \\
\text { lumbar spine }\end{array}$ & $54(34.4)$ & $54(31.0)$ & \\
\hline $\begin{array}{l}\text { Spondylolysis and } \\
\text { spondylolisthesis }\end{array}$ & $2(1.3)$ & $8(4.6)$ & \\
\hline $\begin{array}{l}\text { Compression fracture } \\
\text { due to osteoporosis }\end{array}$ & $3(1.9)$ & I (0.6) & \\
\hline Other & $27(17.2)$ & $30(17.2)$ & \\
\hline $\begin{array}{l}\text { Duration of pain, months, } \\
\text { mean }\end{array}$ & $73.8 \pm 81.4$ & $85.1 \pm 97.8$ & 0.265 \\
\hline S-LANSS, mean \pm SD & $9.1 \pm 6.2$ & $9.6 \pm 5.9$ & 0.513 \\
\hline $\begin{array}{l}\text { Pain medication use, } \\
\mathrm{n}(\%)\end{array}$ & $157(100)$ & $174(100)$ & - \\
\hline $\begin{array}{l}\text { Nonsteroidal anti- } \\
\text { inflammatory drugs }\end{array}$ & $152(96.8)$ & $166(95.4)$ & 0.509 \\
\hline $\begin{array}{l}\text { Anti-depressive drugs } \\
\text { for CLBP }\end{array}$ & $3(1.9)$ & $3(1.7)$ & 0.899 \\
\hline $\begin{array}{l}\text { Anti-epileptic drugs } \\
\text { for CLBP }\end{array}$ & 0 & 0 & - \\
\hline Weak opioids & $15(9.6)$ & $20(11.5)$ & 0.567 \\
\hline Strong opioids & I (0.6) & I $(0.6)$ & 0.942 \\
\hline Other & $53(33.8)$ & $44(25.3)$ & 0.091 \\
\hline PRSIS, mean \pm SD & $3.1 \pm 2.7$ & $2.9 \pm 2.4$ & 0.409 \\
\hline Pain NRS $\pm S D$ & $6.3 \pm 1.2$ & $5.8 \pm 1.1$ & $<0.00$ I \\
\hline $\mathrm{RMDQ} \pm \mathrm{SD}$ & $10.6 \pm 5.2$ & $9.0 \pm 4.9$ & 0.006 \\
\hline $\begin{array}{l}\text { EQ-5D-5L VAS, } \\
\text { mean } \pm \text { SD }^{b}\end{array}$ & $60.7 \pm 17.7$ & $62.9 \pm 16.3$ & 0.245 \\
\hline $\begin{array}{l}\text { EQ-5D-5L quality of life } \\
\text { score, mean } \pm \text { SD }\end{array}$ & $0.6585 \pm 0.174 \mid$ & $0.7242 \pm 0.1515$ & $<0.001$ \\
\hline
\end{tabular}

Notes: aStudent's t-test and chi-square test for continuous and categorical data, respectively; 'standardized to a $0-100$ scale.

Abbreviations: EQ-5D-5L VAS, 5-level, 5 dimension EuroQol visual analog scale; NRS, numerical rating scale; PRSIS, Pain-Related Sleep Interference Scale; RMDQ, Roland-Morris Disability Questionnaire; SD standard deviation; CLBP, chronic low back pain.

$0.0600 ; P=0.017$ ), but not at week 8 (LS mean difference $0.0204,95 \%$ CI $-0.0091,0.0499 ; P=0.175$ ).

The majority of patients treated with pregabalin achieved global improvements from both the clinician and patient perspectives, $80.8 \%$ and $74.4 \%$ for the CGI-C and PGI-C, respectively (Figure 5). In contrast, less than half of the patients in usual care achieved global improvements
(Figure 5). The differences between treatment groups across all seven change categories were statistically significant on both the CGI-C and PGI-C (both $P<0.001$ ).

Sensitivity analysis performed for the primary endpoint, PRSIS at week 8, in which missing values were imputed using BOCF showed results that were consistent in direction and comparable magnitude with the primary analysis; LS mean changes from baseline were -1.1 and -0.3 for pregabalin and usual care, respectively, with a difference between treatments of -0.7 ( $95 \% \mathrm{CI}-1.2,-0.3 ; P=0.002)$.

\section{Safety}

In the pregabalin group, 56 treatment-related AEs were reported by 40 patients $(25.5 \%)$, of which none was serious (Table 2). Treatment-related AEs was the reason for discontinuation in $9.6 \%$ of the pregabalin-treated patients. The most commonly reported treatment-related AEs in the pregabalin group were somnolence (13.4\%) and dizziness (10.8\%) (Table 2).

\section{Discussion}

The results of this non-interventional study of CLBP-NeP patients treated with pregabalin showed significant improvements in pain-related sleep interference, pain, function, and health status relative to usual care. These improvements were not only observed at the primary endpoint of 8 weeks, but most also showed significance after 4 weeks of treatment. Pregabalin was also safe and well tolerated. Dosing in this study was flexible, reflecting real-world clinical practice, and the dose range was $25-300 \mathrm{mg} /$ day; the recommended dose range of pregabalin in Japan is $150-600 \mathrm{mg} /$ day.

In addition to improvements in sleep, pregabalin resulted in greater benefits in pain and function relative to usual care at both 4 and 8 weeks, manifested by significantly greater reductions in pain NRS scores and RMDQ scores, respectively. Of note, the $38 \%$ change in RMDQ at week 8 exceeded the minimal clinically important difference of $30 \%$, and showed that this change was clinically relevant as well as statistically significant. However, the results for pain are in contrast to a small randomized, cross-over trial in patients with a related condition, neurogenic claudication associated with lumbar spinal stenosis that reported no effects of pregabalin on increasing the time to moderate pain during a treadmill test. ${ }^{63}$ This difference in analgesic efficacy may be a function of the type of outcomes assessed as well as the patient populations.

The significant improvements in sleep and pain are not surprising given the reciprocal relationship between 


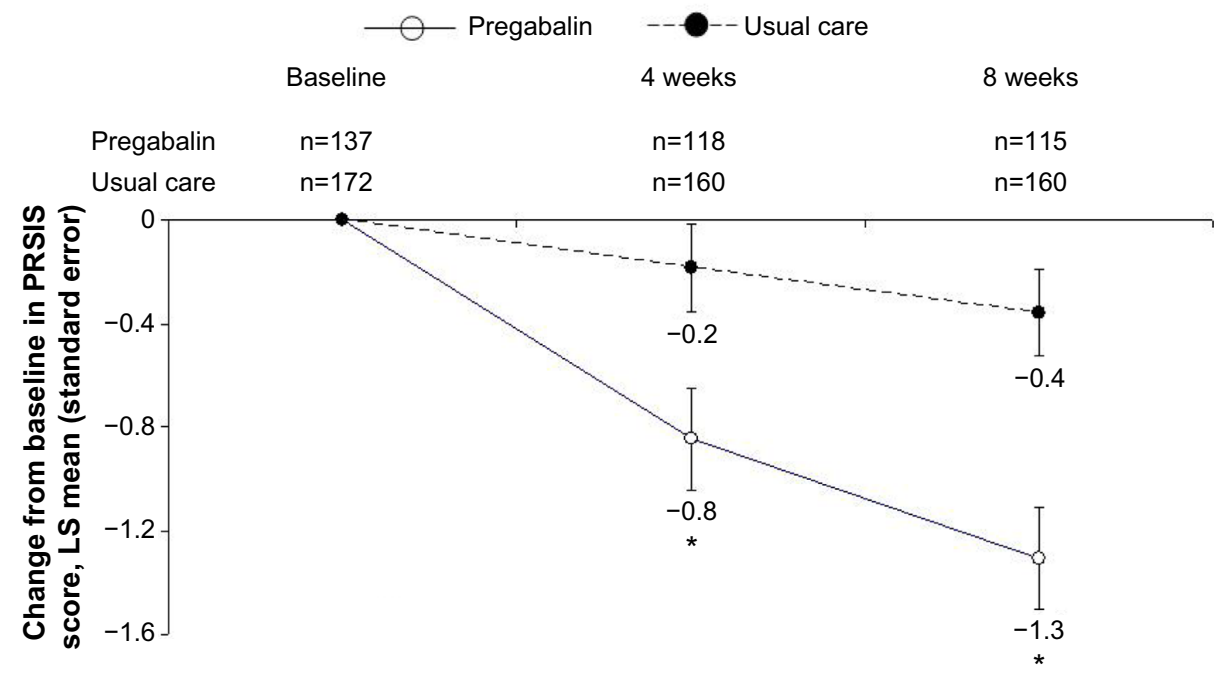

Figure 2 Change from baseline in sleep disturbance, assessed using the Pain-Related Sleep Interference Scale (PRSIS).

Note: $* P<0.05$ versus usual care.

Abbreviation: LS, least-squares.

these two outcomes such that not only does pain interfere with sleep but poor sleep increases pain sensitivity. ${ }^{16,17,64,65}$ Observations from previous studies of pregabalin in several $\mathrm{NeP}$ conditions also showed significant effects on both pain and pain-related sleep interference. ${ }^{31-33,51,66,67}$ Additionally, it has been suggested that improvements in function and health state in patients treated with pregabalin are not exclusively mediated through analgesic efficacy, but rather result from combined effects on pain and sleep interference. ${ }^{68}$ Those observations provide support for the current results, which

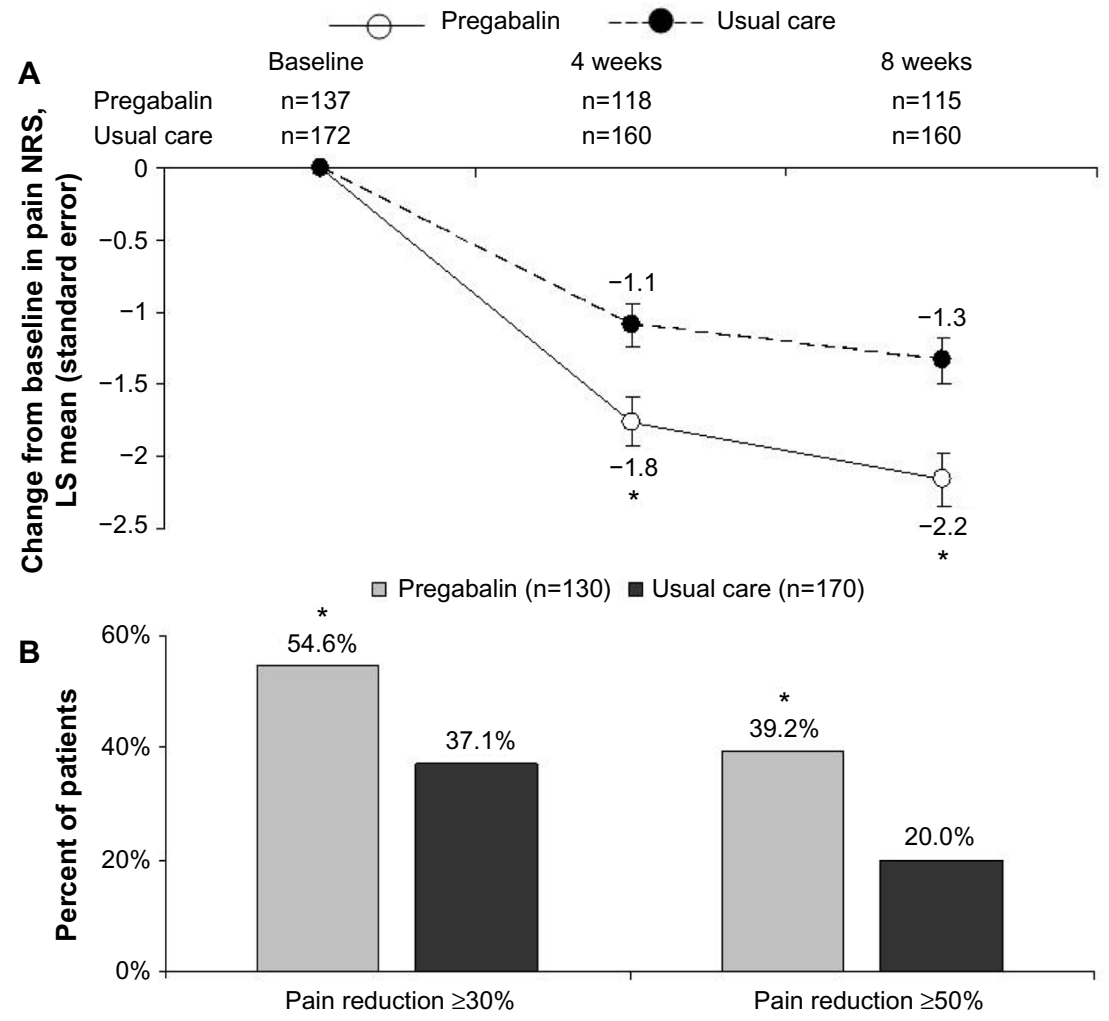

Figure 3 Efficacy of treatment for pain assessed using a pain numerical rating scale (NRS).

Notes: (A) Change from baseline. (B) Proportions of patients at week 8 (last observation carried forward) reporting clinically relevant improvements in pain. $* P<0.05$ versus usual care.

Abbreviation: LS, least-squares. 


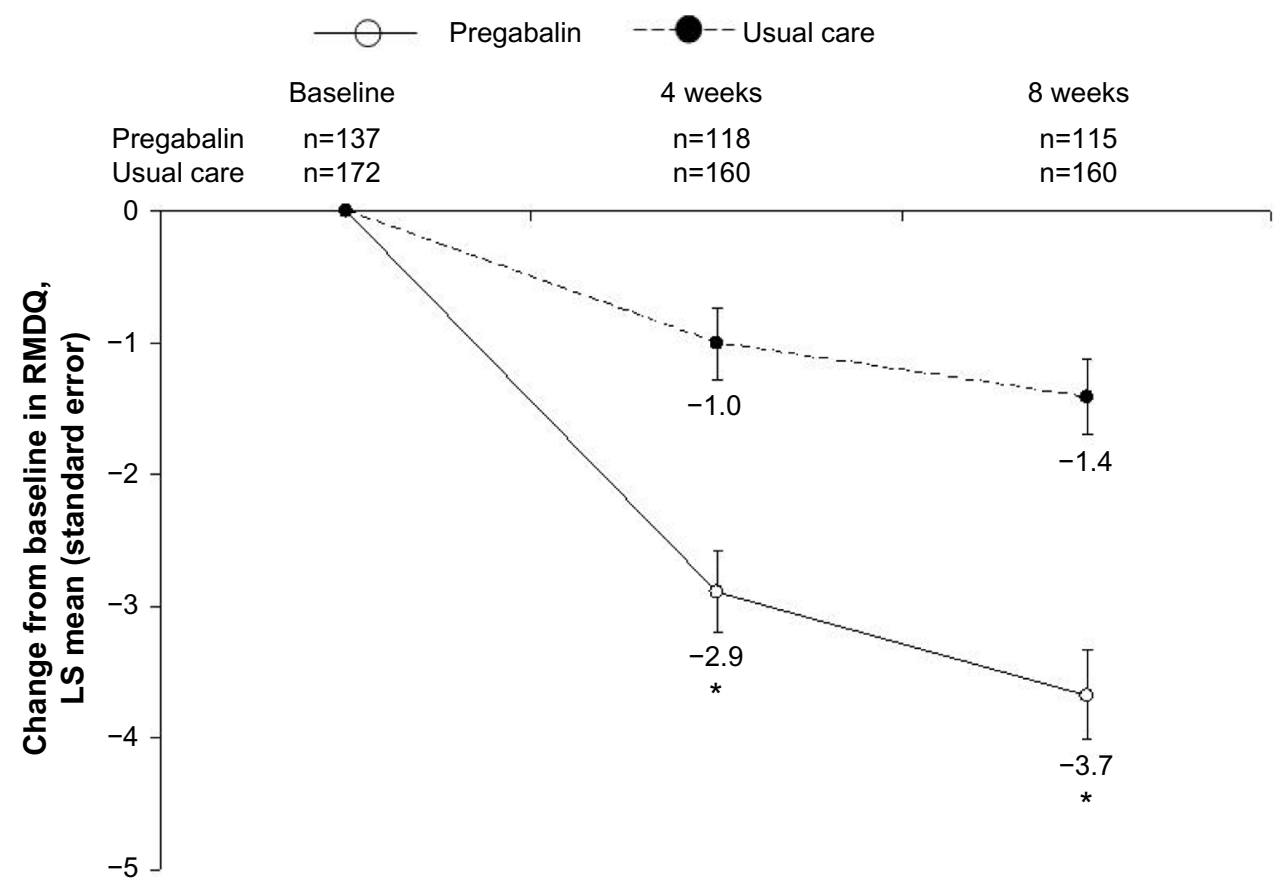

Figure 4 Change from baseline in function, assessed by the Roland-Morris Disability Questionnaire (RMDQ).

Note: $* P<0.05$ versus usual care.

Abbreviation: LS, least-squares.

showed that the benefits achieved with pregabalin on pain and sleep may be further manifested by improvements in overall health state, as measured by the EQ-5D-5L VAS scores and the global impression of change scores. Notably, there was good concordance between CGI-C and PGI-C, further suggesting the robustness of these changes.
All patients in both treatment groups had at least moderate levels of pain, which was an inclusion criterion. These levels of pain, despite previous use of analgesic therapies, highlight the refractoriness of CLBP with $\mathrm{NeP}$ as well as the complexity of its treatment. However, it should also be noted that patients who received pregabalin had significantly

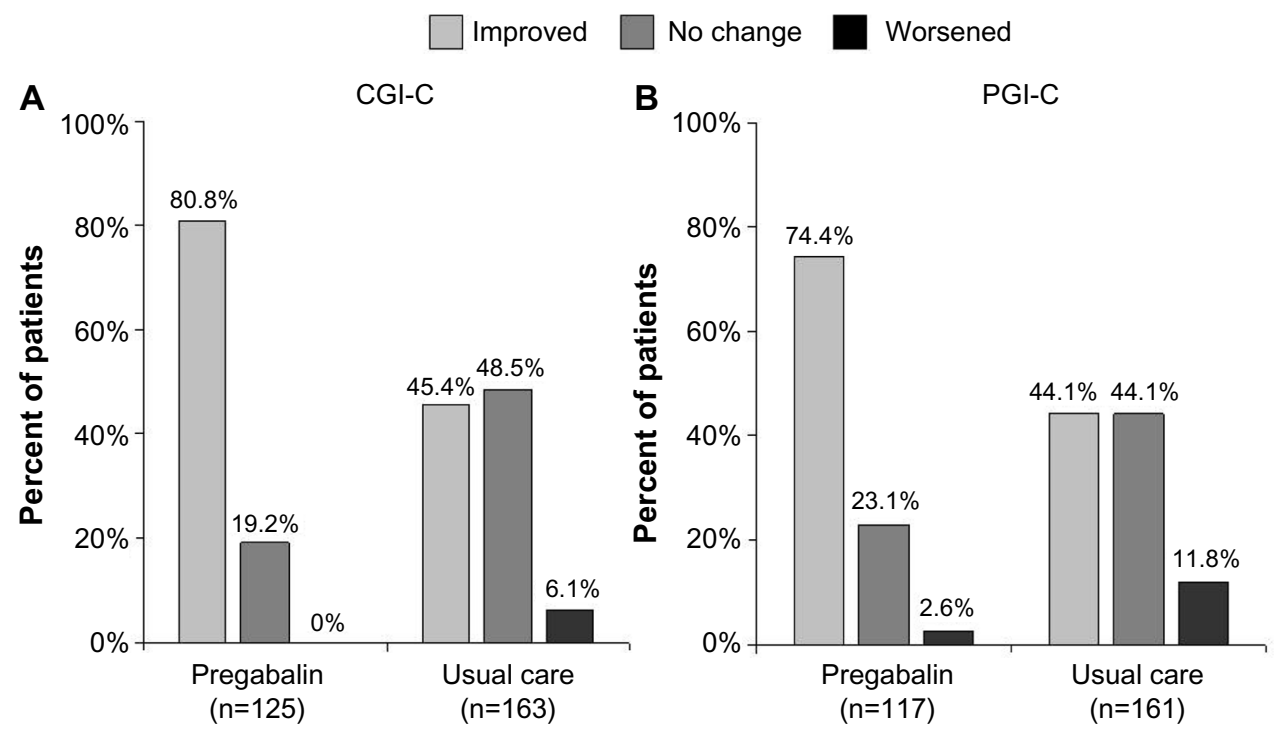

Figure 5 Global impression of change at week 8 from the perspectives of $(\mathbf{A})$ the clinician, assessed using the Clinician Global Impression of Change (CGI-C), and (B) the patient, assessed using the Patient Global Impression of Change (PGI-C).

Notes: Categories are collapsed into "Improved", consisting of "minimally improved", "much improved", and "very much improved", and "Worsened", consisting of "minimally worse", "much worse", and "very much worse". Differences between pregabalin and usual care were significant for the full set of categories on the CGI-C and PGI-C (both $P<0.05)$. 
Table 2 Treatment-related adverse events (AEs) in the pregabalin group $(n=157)$

\begin{tabular}{|c|c|}
\hline Treatment-related AE & Incidence, $\mathbf{n}(\%)$ \\
\hline Number of treatment-related AEs & 56 \\
\hline Patients with any treatment-related AEs & $40(25.5)$ \\
\hline Patients with serious treatment-related AEs & 0 \\
\hline $\begin{array}{l}\text { Treatment discontinuations due to treatment- } \\
\text { related AEs }\end{array}$ & $15(9.6)$ \\
\hline $\begin{array}{l}\text { Patients with dose reduced or temporary } \\
\text { discontinuation due to treatment-related AEs }\end{array}$ & $21(13.4)$ \\
\hline \multicolumn{2}{|c|}{ Most common treatment-related AEs occurring in $\geq 3 \%$ of patients } \\
\hline Somnolence & $21(13.4)$ \\
\hline Dizziness & $17(10.8)$ \\
\hline
\end{tabular}

greater pain scores at baseline as well as greater functional impairment on the RMDQ. These higher pain scores were similar to another non-interventional study in CLBP-NeP patients that also reported significantly greater baseline pain among those treated with pregabalin relative to usual care. ${ }^{61}$ The consistency of the higher baseline pain scores suggests that, although pregabalin is effective for the treatment of $\mathrm{NeP}$, clinicians may be reserving its use in the clinical setting for patients with more refractory NeP.

Consistent with the safety profile that has been reported in other studies of pregabalin for NeP in Japan, ${ }^{34,69}$ pregabalin was safe and well tolerated. There were no serious AEs, and only $9.6 \%$ of patients discontinued the study due to treatment-related AEs. No patients in the comparator group discontinued due to AEs, which may be accounted for by the fact that physicians were allowed to switch patients to other analgesics throughout the study (ie, real-world prescribing). The low rate of study discontinuations among the usual care patients, especially related to AEs, may have been due, at least in part, to the ability to switch medications throughout the study based on safety, efficacy, and the treating physician's judgment. In contrast, those in the pregabalin arm, although also able to switch among other analgesics, were required to remain on pregabalin through week 8 . It should also be considered that the higher proportion of pregabalin patients who discontinued due to being lost to follow-up may have stopped their clinic visits as a result of improvement. Such a reason for lost to follow-up has previously been reported in other conditions, ${ }^{70-72}$ suggesting that these discontinuations are not necessarily associated with adverse outcomes. However, even if subjects who discontinued had no benefit from the treatment, which is the most conservative approach for handling missing value, results of BOCF were similar to that of the primary analysis, thus supporting the robustness of the effects.
Several study limitations should be noted, including that the study was observational and open-label as well as nonrandomized, since choice of treatment was based on the clinical judgment of the treating physician. This design may also be considered as setting an imbalance in clinical equipoise, since the known effects of pregabalin on the part of the clinicians may have biased patient selection, subsequently resulting in the significantly greater pain observed in this group. Nevertheless, this design can also be considered as a strength of the study, since it allows a physician to assess a drug's efficacy and safety in real-world clinical practice, which does not itself endorse balanced treatment strategies.

Patients in this study were required to have at least a moderate level of pain that was refractory to analgesics. The use of these inclusion criteria may also limit the generalizability of the results. However, greater pain, rather than greater sleep disturbance, may more likely result in the type of clinical decisions and treatment used in the current analysis.

\section{Conclusion}

The present study adds to previous studies of pregabalin for $\mathrm{NeP}$ by providing clinically important information on patients with CLBP-NeP, a difficult to treat population, in a usual care setting. When used under conditions of real-world prescribing for the treatment of CLBP-NeP, pregabalin, which could be used either as monotherapy or in combination with usual care prescribing, showed significantly greater efficacy than the usual care alone group in reducing pain-related sleep interference as well as the pain itself. Improvements in sleep were accompanied by significant improvement in pain, function, and health status, all of which contribute to the significantly improved quality of life in patients with CLBP-NeP. In addition, the majority of patients showed good tolerance of pregabalin. These results provide evidence of the clinical benefit of pregabalin and confirm its usefulness for treating patients with this challenging condition.

\section{Acknowledgment}

Editorial assistance was provided by E Jay Bienen, who was funded by Pfizer. We would like to extend our thanks to all of the participating physicians and patients involved in this study.

\section{Disclosure}

This study was sponsored by Pfizer. Stephen Watt, Bruce Parsons, Alesia Sadosky, Kazutaka Nozawa, Kazuhiro Hayakawa, Tamotsu Yoshiyama, Nozomi Ebata, and Koichi Fujii are employees and shareholders of Pfizer, the sponsor of this study. None of the other authors were financially 
compensated for their collaboration in this project or for the development of this paper.

\section{References}

1. Vos T, Flaxman AD, Naghavi M, et al. Years lived with disability (YLDs) for 1160 sequelae of 289 diseases and injuries 1990-2010: a systematic analysis for the Global Burden of Disease Study 2010. Lancet. 2012;380(9859):2163-2196.

2. Dagenais S, Caro J, Haldeman S. A systematic review of low back pain cost of illness studies in the United States and internationally. Spine J. 2008;8(1):8-20.

3. Stewart WF, Ricci JA, Chee E, Morganstein D, Lipton R. Lost productive time and cost due to common pain conditions in the US workforce. JAMA. 2003;290(18):2443-2454.

4. Fujii T, Matsudaira K. Prevalence of low back pain and factors associated with chronic disabling back pain in Japan. Eur Spine J. 2013; 22(2):432-438.

5. Suka M, Yoshida K. Low back pain deprives the Japanese adult population of their quality of life: a questionnaire survey at five healthcare facilities in Japan. Environ Health Prev Med. 2008;13(2):109-115.

6. Sadosky AB, DiBonaventura M, Cappelleri JC, Ebata N, Fujii K. The association between lower back pain and health status, work productivity, and health care resource use in Japan. J Pain Res. 2015;8: 119-130.

7. Becker A, Held H, Redaelli M, et al. Low back pain in primary care. Costs of care and prediction of future health care utilization. Spine (Phila Pa 1976). 2010;35(18):1714-1720.

8. Freynhagen R, Baron R. The evaluation of neuropathic components in low back pain. Curr Pain Headache Rep. 2009;13(3):185-190.

9. Giesecke T, Gracely RH, Grant MA, et al. Evidence of augmented central pain processing in idiopathic chronic low back pain. Arthritis Rheum. 2004;50(2):613-623.

10. Dionne CE, Dunn KM, Croft PR, et al. A consensus approach toward the standardization of back pain definitions for use in prevalence studies. Spine (Phila Pa 1976). 2008;33(1):95-103.

11. Webb R, Brammah T, Lunt M, Urwin M, Allison T, Symmons D. Prevalence and predictors of intense, chronic, and disabling neck and back pain in the UK general population. Spine (Phila Pa 1976). 2003; 28(11):1195-1202.

12. Yamashita T, Takahashi K, Yonenobu K, Kikuchi S. Prevalence of neuropathic pain in cases with chronic pain related to spinal disorders. J Orthop Sci. 2014;19(1):15-21.

13. Freynhagen R, Baron R, Gockel U, Tolle TR. painDETECT: a new screening questionnaire to identify neuropathic components in patients with back pain. Curr Med Res Opin. 2006;22(10):1911-1920.

14. Fishbain DA, Cole B, Lewis JE, Gao J. What is the evidence that neuropathic pain is present in chronic low back pain and soft tissue syndromes? An evidence-based structured review. Pain Med. 2014; 15(1):4-15.

15. Ogawa S, Komatsu M, Ohno S, Yamane H, Hayakawa K. [Interim report of drug use investigations of pregabalin (Lyrica)]. Progress in Medicine. 2013;33(10):2159-2171. Japanese.

16. McCracken LM, Iverson GL. Disrupted sleep patterns and daily functioning in patients with chronic pain. Pain Res Manag. 2002;7(2):75-79.

17. Smith MT, Haythornthwaite JA. How do sleep disturbance and chronic pain inter-relate? Insights from the longitudinal and cognitive-behavioral clinical trials literature. Sleep Med Rev. 2004;8(2):119-132.

18. Kundermann B, Lautenbacher S. Effects of impaired sleep quality and sleep deprivation on diurnal pain perception. In: Lavigne G, Sessle B, Choiniere M, Soja P, editors. Sleep and Pain. Seattle: IASP Press; 2007:137-152.

19. Kelly GA, Blake C, Power CK, O'Keeffe D, Fullen BM. The association between chronic low back pain and sleep: a systematic review. Clin J Pain. 2011;27(2):169-181.

20. Marin R, Cyhan T, Miklos W. Sleep disturbance in patients with chronic low back pain. Am J Phys Med Rehabil. 2006;85(5):430-435.
21. Marty M, Rozenberg S, Duplan B, et al. Quality of sleep in patients with chronic low back pain: a case-control study. Eur Spine J. 2008; 17(6):839-844.

22. Mann R, Bergstrom F, Schaefer C, et al. Burden of spinal cord injuryrelated neuropathic pain in the United States: retrospective chart review and cross-sectional survey. Spinal Cord. 2013;51(7):564-570.

23. Langley PC, Van Litsenburg C, Cappelleri JC, Carroll D. The burden associated with neuropathic pain in Western Europe. J Med Econ. 2013; 16(1):85-95.

24. Sadosky A, Schaefer C, Mann R, et al. Burden of chronic low back pain with a neuropathic pain component: retrospective chart review and cross-sectional survey among adults seeking treatment in the United States. J Pain Relief. 2014;3:163.

25. Roland M, Morris R. A study of the natural history of back pain. Part I: Development of a reliable and sensitive measure of disability in low back pain. Spine (Phila Pa 1976). 1983;8(2):141-144.

26. The Japanese Orthopaedic Association. [The practice guideline for low back pain 2012]. Nankodo Co, Ltd, Tokyo, Japan. Available from: http://minds.jcqhc.or.jp/n/medical_user_main.php. Accessed July 7, 2015. Japanese.

27. National Institute for Health and Clinical Excellence [homepage on the Internet]. Neuropathic pain - pharmacological management: The pharmacological management of neuropathic pain in adults in non-specialist settings. NICE; 2013. Available from: http://www.nice.org.uk/guidance/ cg173/chapter/1-recommendations. Accessed April 27, 2015.

28. Koes BW, van Tulder M, Lin CW, Macedo LG, McAuley J, Maher C. An updated overview of clinical guidelines for the management of non-specific low back pain in primary care. Eur Spine J. 2010;19(12): 2075-2094.

29. Dworkin RH, O'Connor AB, Audette J, et al. Recommendations for the pharmacological management of neuropathic pain: an overview and literature update. Mayo Clin Proc. 2010;85(3 Suppl):S3-S14.

30. Field MJ, Cox PJ, Stott E, et al. Identification of the alpha2-delta-1 subunit of voltage-dependent calcium channels as a molecular target for pain mediating the analgesic actions of pregabalin. Proc Natl Acad Sci U S A. 2006;103(46):17537-17542.

31. Satoh J, Yagihashi S, Baba M, et al. Efficacy and safety of pregabalin for treating neuropathic pain associated with diabetic peripheral neuropathy: a 14 week, randomized, double-blind, placebo-controlled trial. Diabet Med. 2011;28(1):109-116.

32. Ogawa S, Suzuki M, Arakawa A, et al. [Evaluation of the efficacy and safety of pregabalin in the treatment of postherpetic neuralgia: a randomized, double-blind, multicenter, placebo-controlled study]. J Japan Soc Pain Clin. 2010;17(2):141-152. Japanese.

33. Cardenas DD, Nieshoff EC, Suda K, et al. A randomized trial of pregabalin in patients with neuropathic pain due to spinal cord injury. Neurology. 2013;80(6):533-539.

34. Ogawa S, Satoh J, Arakawa A, Yoshiyama T, Suzuki M. Pregabalin treatment for peripheral neuropathic pain: a review of safety data from randomized controlled trials conducted in Japan and in the west. Drug Saf. 2012;35(10):793-806.

35. Roth T, Arnold LM, Garcia-Borreguero D, Resnick M, Clair AG. A review of the effects of pregabalin on sleep disturbance across multiple clinical conditions. Sleep Med Rev. 2014;18(3):261-271.

36. Toelle T, Varvara R, Nimour M, Emir B, Brasser M. Pregabalin in neuropathic pain related to DPN, cancer and back pain: analysis of a 6-week observational study. Open Pain J. 2012;5:1-11.

37. Saldana MT, Navarro A, Perez C, Masramon X, Rejas J. Patient-reportedoutcomes in subjects with painful lumbar or cervical radiculopathy treated with pregabalin: evidence from medical practice in primary care settings. Rheumatol Int. 2010;30(8):1005-1015.

38. Goren A, Mould-Quevedo J, daCosta DiBonaventura M. Prevalence of pain reporting and associated health outcomes across emerging markets and developed countries. Pain Med. 2014;15(11):1880-1891.

39. Turk DC, Dworkin RH, Revicki D, et al. Identifying important outcome domains for chronic pain clinical trials: an IMMPACT survey of people with pain. Pain. 2008;137(2):276-285. 
40. Turk DC, Dworkin RH, Allen RR, et al. Core outcome domains for chronic pain clinical trials: IMMPACT recommendations. Pain. 2003;106(3):337-345.

41. Dworkin RH, Turk DC, Farrar JT, et al. Core outcome measures for chronic pain clinical trials: IMMPACT recommendations. Pain. 2005;113(1-2):9-19.

42. Haanpaa M, Attal N, Backonja M, et al. NeuPSIG guidelines on neuropathic pain assessment. Pain. 2011;152(1):14-27.

43. Bennett MI, Smith BH, Torrance N, Potter J. The S-LANSS Score for identifying pain of predominantly neuropathic origin: validation for use in clinical and postal research. J Pain. 2005;6(3):149-158.

44. Smith MT, Edwards RR, McCann UD, Haythornthwaite JA. The effects of sleep deprivation on pain inhibition and spontaneous pain in women. Sleep. 2007;30(4):494-505.

45. Smith MT, Perlis ML, Smith MS, Giles DE, Carmody TP. Sleep quality and presleep arousal in chronic pain. J Behav Med. 2000;23(1):1-13.

46. Morin CM, Gibson D, Wade J. Self-reported sleep and mood disturbance in chronic pain patients. Clin J Pain. 1998;14(4):311-314.

47. Finan PH, Goodin BR, Smith MT. The association of sleep and pain: an update and a path forward. J Pain. 2014;14(12):1539-1552.

48. Sezgin M, Hasanefendioglu EZ, Ali Sungur M, et al. Sleep quality in patients with chronic low back pain: A cross-sectional study assesing its relations with pain, functional status and quality of life. $J$ Back Musculoskelet Rehabil. Epub October 15, 2014.

49. Cleeland CS, Ryan KM. Pain assessment: global use of the Brief Pain Inventory. Ann Acad Med Singapore. 1994;23(2):129-138.

50. Vernon MK, Brandenburg NA, Alvir JM, Griesing T, Revicki DA. Reliability, validity, and responsiveness of the daily sleep interference scale among diabetic peripheral neuropathy and postherpetic neuralgia patients. J Pain Symptom Manage. 2008;36(1):54-68.

51. Anastassiou E, Iatrou CA, Vlaikidis N, et al. Impact of pregabalin treatment on pain, pain-related sleep interference and general well-being in patients with neuropathic pain: a non-interventional, multicentre, post-marketing study. Clin Drug Investig. 2011;31(6):417-426.

52. Parsons B, Emir B, Knapp L. Examining the Time to Improvement of Sleep Interference With Pregabalin in Patients With Painful Diabetic Peripheral Neuropathy and Postherpetic Neuralgia. Am J Ther. Epub September 30, 2014.

53. Ostelo RW, de Vet HC. Clinically important outcomes in low back pain. Best Pract Res Clin Rheumatol. 2005;19(4):593-607.

54. Ostelo RW, Deyo RA, Stratford P, et al. Interpreting change scores for pain and functional status in low back pain. Towards international consensus regarding minimal important change. Spine (Phila Pa 1976). 2008; 33(1):90-94.

55. Jordan K, Dunn KM, Lewis M, Croft P. A minimal clinically important difference was derived for the Roland-Morris Disability Questionnaire for low back pain. J Clin Epidemiol. 2006;59(1):42-52.

56. Dworkin RH, Turk DC, Wyrwich KW, et al. Interpreting the clinical importance of treatment outcomes in chronic pain clinical trials: IMMPACT recommendations. J Pain. 2008;9(2):105-121.

57. Herdman M, Gudex C, Lloyd A, et al. Development and preliminary testing of the new five-level version of EQ-5D (EQ-5D-5L). Qual Life Res. 2011;20(10):1727-1736.

Journal of Pain Research

\section{Publish your work in this journal}

The Journal of Pain Research is an international, peer-reviewed, open access, online journal that welcomes laboratory and clinical findings in the fields of pain research and the prevention and management of pain. Original research, reviews, symposium reports, hypothesis formation and commentaries are all considered for publication.
58. Guy W. ECDEU assessment manual for psychopharmacology, revised. US Department of Health, Education, and Welfare publication (ADM). Rockville, MD: National Institute of Mental Health; 1976.

59. Ikeda S, Shiroiwa T, Igarashi A, et al. [Developing a Japanese version of the EQ-5D-5L value set]. J Natl Inst Public Health. 2015;64(1):47-55. Japanese.

60. Suzukamo Y, Fukuhara S, Kikuchi S, et al. Validation of the Japanese version of the Roland-Morris Disability Questionnaire. J Orthop Sci. 2003;8(4):543-548.

61. Morera-Dominguez C, Ceberio-Balda F, Florez-Garcia M, Masramon X, Lopez-Gomez V. A cost-consequence analysis of pregabalin versus usual care in the symptomatic treatment of refractory low back pain: sub-analysis of observational trial data from orthopaedic surgery and rehabilitation clinics. Clin Drug Investig. 2010; 30(8):517-531.

62. Mallinckrodt CH, Lane PW, Schnell D, Peng Y, Mancuso JP. Recommendations for the primary analysis of continuous endpoints in longitudinal clinical trials. Drug Inf J. 2008;42(4):303-319.

63. Markman JD, Frazer ME, Rast SA, et al. Double-blind, randomized, controlled, crossover trial of pregabalin for neurogenic claudication. Neurology. 2015;84(3):265-272.

64. Affleck G, Urrows S, Tennen H, Higgins P, Abeles M. Sequential daily relations of sleep, pain intensity, and attention to pain among women with fibromyalgia. Pain. 1996;68(2-3):363-368.

65. Edwards RR, Almeida DM, Klick B, Haythornthwaite JA, Smith MT. Duration of sleep contributes to next-day pain report in the general population. Pain. 2008;137(1):202-207.

66. Roth T, van Seventer R, Murphy TK. The effect of pregabalin on pain-related sleep interference in diabetic peripheral neuropathy or postherpetic neuralgia: a review of nine clinical trials. Curr Med Res Opin. 2010;26(10):2411-9241.

67. Vinik A, Emir B, Parsons B, Cheung R. Prediction of pregabalinmediated pain response by severity of sleep disturbance in patients with painful diabetic neuropathy and post-herpetic neuralgia. Pain Med. 2014;15(4):661-670.

68. Vinik A, Emir B, Cheung R, Whalen E. Relationship between pain relief and improvements in patient function/quality of life in patients with painful diabetic peripheral neuropathy or postherpetic neuralgia treated with pregabalin. Clin Ther. 2013;35(5):612-623.

69. Onouchi K, Koga H, Yokoyama K, Yoshiyama T. An open-label, long-term study examining the safety and tolerability of pregabalin in Japanese patients with central neuropathic pain. J Pain Res. 2014;7: $439-447$.

70. Joshi AB, Gill GS, Smith PL. Outcome in patients lost to follow-up. J Arthroplasty. 2003;18(2):149-153.

71. Poon KH, Yeo S, Tong L. Lost to follow-up for appointments in a dedicated dry eye clinic. Patient Prefer Adherence. 2014;8:1409-1418.

72. Han JJ, Lee JH, Oh SH, Chang SO, Suh MW. Assessing the Effects of Tinnitus Retraining Therapy in Patients Lost to Follow-up: A Telephone Survey. Otol Neurotol. 2015;36(4):581-587.

\section{Dovepress}

The manuscript management system is completely online and includes a very quick and fair peer-review system, which is all easy to use. Visit http://www.dovepress.com/testimonials.php to read real quotes from published authors. 